EPJ Web of Conferences 33, 02012 (2012)

DOI: $10.1051 /$ epjconf/20123302012

(C) Owned by the authors, published by EDP Sciences, 2012

\title{
Structural Engineering of Vacancy Defected Bismuth Tellurides for Thermo-electric Applications
}

\author{
K. Termentzidis ${ }^{1, a}$, A. Pokropivny ${ }^{1}$, S.-Y. Xiong ${ }^{1}$, Y. Chumakov $^{1,2}$, P. Cortona ${ }^{3}$ and S. Volz ${ }^{1}$ \\ ${ }^{1}$ Laboratoire d'Energétique Moléculaire et Macroscopique, Combustion, UPR CNRS 288 Ecole \\ Centrale Paris, 92295, Châtenay-Malabry, France \\ ${ }^{2}$ Institute of Applied Physics of the Academy of Sciences of Moldova, MD 2028, Kishinev, \\ Akademicheskaya 5, Moldova \\ ${ }^{3}$ Laboratoire Structures, Propriétés et Modélisation des Solides, UMR CNRS 8580, École Centrale \\ Paris, 92295, Châtenay-Malabry, France
}

\begin{abstract}
Molecular Dynamics and ab-initio simulations are used to find the most stable stoichiometries of Bismuth Tellurides with vacancy defects. The interest is to decrease the thermal conductivity of these compounds a key point to achieve high figure of merits. A reduction of $70 \%$ of the thermal conductivity is observed with Te vacancies of only $5 \%$.
\end{abstract}

\section{Introduction}

At the room temperature Bismuth Telluride is the best thermoelectric material with figure of merit near to unity. Due to its better thermoelectric figure of merit at room temperature, bismuth telluride and some related ternary alloys (with antimony or selenium) are often used for cooling applications in commercial Peltier elements. The key point to increase the figure of merit is to create structural defects with purpose to decrease the thermal conductivity. The lattice thermal conductivity that is predominant for semiconducting materials depends strongly on the additional phonon scattering. This scattering can be increased with introducing in the lattice an alloy disordering, a vacancy or substitution defects, or even decrease the dimensions of the lattice (thin films or nanowires). In the current study the vacancy defects are studied. The percentage of vacancies of 1 to $10 \%$ of Bismuth and 1 to $6 \%$ of Tellurium atoms is examined.

The prediction of the thermal conductivity of $\mathrm{Bi}_{2} \mathrm{Te}_{3}$ is made by NEMD method. This method also mentioned as direct method is similar to the hot-plate and cold-plate experimental setups, proposed by Kotake and Wakuri [1], and Amrit et al. [2], respectively. In this method, the heat flux is imposed along the $\mathrm{z}$ direction. Periodic boundary conditions are used in all directions. The planar slab heat source and heat sink are located at the $1 / 4$ th and 3/4th of the simulation cell. With periodic boundary conditions employed, the resulting steady state temperature profile is symmetric according to the positions of the two thermostats that are perpendicular to the z-direction. After reaching the steady state, the temperature profile and the heat power of the two thermostats are collected over several millions time steps, in order to achieve a good statistical accuracy. Then the thermal

\footnotetext{
a e-mail : konstantinos.termentzidis@ecp.fr
} 
conductivity is determined by using the Fourier's law. This method has been used widely for the study of the heat transfer in the nanoscale $[3,4,5,6,7]$. The equilibrium molecular dynamics has also been employed with success $[8,9,4,6]$, but the comparison of the two methods is not in the scope of this article.

\section{Results, Thermal Conductivity}

In MD simulations, we use the two-body atomic Morse potential developed by Qiu and Ruan for its correct description of the thermal properties [10]. This potential contains two terms, i.e. a shortrange interaction term and a Coulombic term for the description of the long-range electrostatic interactions. The charges for the Coulombic terms that have been used are for Te-type $1=-0.26$, $\mathrm{Bi}=0.38$, and Tetype $2=-0.24$, while the masses are for Te 127.6 a.m.u and for Bi 209.0 a.m.u. The long-range Coulombic interactions are computed with the Ewalds's summation. The hexagonal cell parameters are $\mathrm{a}=\mathrm{b}=4.386 \mathrm{~A}$ and $\mathrm{c}=30.497 \mathrm{~A}$. The unit cell contains 15 atoms, which are distributed in 3 quintuples. Each quintuple has two types of Tellurium atoms, the first one at the edges of the quintuple, which interacts with the Bismuth atoms with ionic nature bonds, while there is a combination of Van der Waals and covalent bonds between the two tellurium of different quintuples, which makes these bonds less strong than the previous ones.

\subsection{Bulk}

The thermal conductivity of the bulk $\mathrm{Bi}_{2} \mathrm{Te}_{3}$ has been calculated for average temperatures of the system of $100 \mathrm{~K}, 200 \mathrm{~K}, 300 \mathrm{~K}, 400 \mathrm{~K}$ and $500 \mathrm{~K}$. The two thermostats are set with a temperature difference of $10 \%$ in comparison to the average temperature of the system. Two system sizes of bulk materials have been simulated, the $8 \times 8 \times 4$ and $8 \times 8 \times 8$ unit cells (Figure 1).

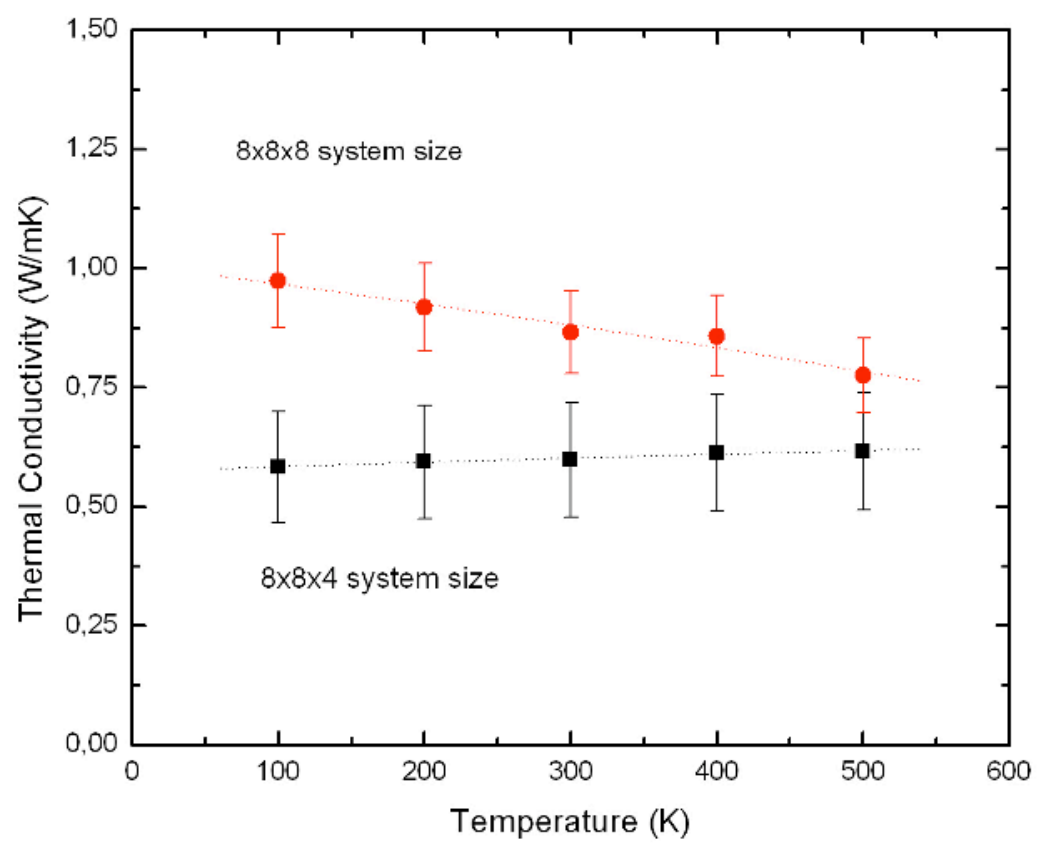

Fig. 1. Thermal conductivity for bulk Bi2Te3 as a function of temperature calculated with NEMD for two system sizes. 
For the bulk $\mathrm{Bi}_{2} \mathrm{Te}_{3}$, we used also the proposed method by Shelling [11] to find the thermal conductivity for infinite size system (figure 2) and to compare it with the experimental results. The thermal conductivity for infinite system size of bulk $\mathrm{Bi}_{2} \mathrm{Te}_{3}$ is plotted as a function of temperature in Figure 2. Two experimental results for $300 \mathrm{~K}$ are reported: $1.57 \mathrm{~W} / \mathrm{mK}$ from Goldsmid [12] and 1.70 $\mathrm{W} / \mathrm{mK}$ from Satterthwaire-Ure [13]. The NEMD method agrees with the experimental results with a very satisfying accuracy. The thermal conductivity of bulk $\mathrm{Bi}_{2} \mathrm{Te}_{3}$ shows temperature dependence typical of that of crystalline solids. At higher temperatures, phonon-phonon scattering, which is the predominant scattering process, shows a $1 / \mathrm{T}$ dependence with temperature. This reduction of thermal conductivity is explained within the Debye model by the increased scattering rate of higher frequency phonons excited at higher temperatures.

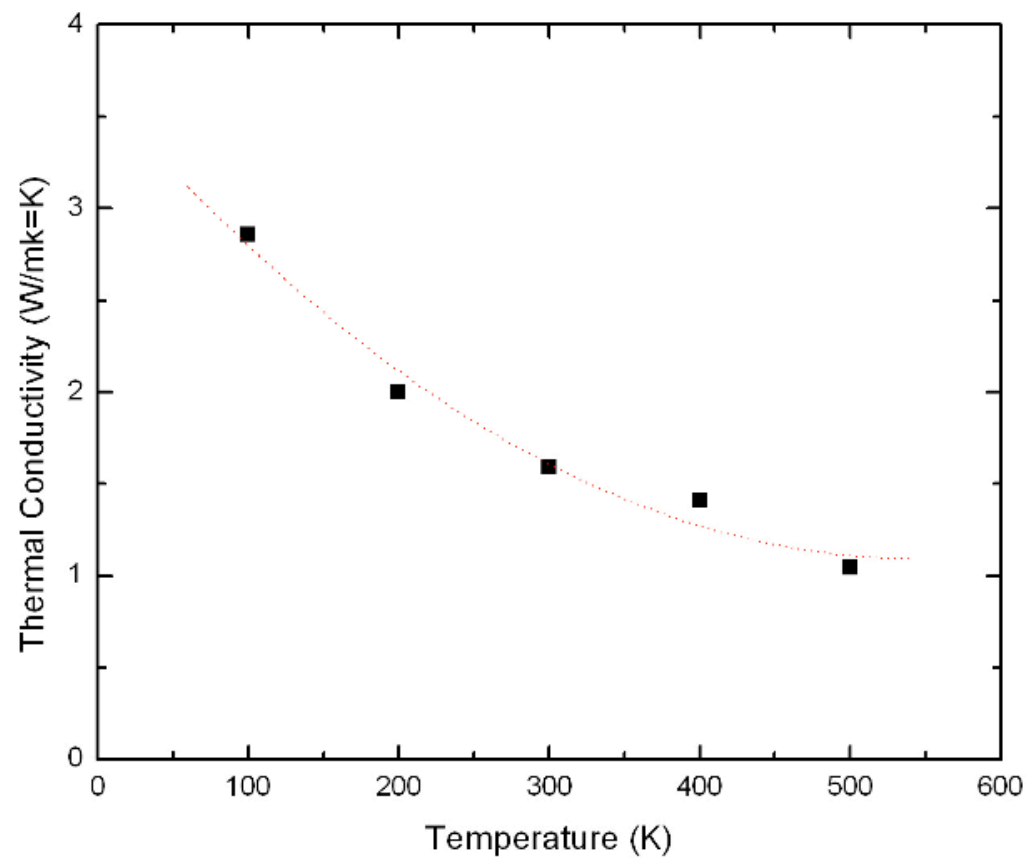

Fig. 2. Thermal conductivity for bulk $\mathrm{Bi}_{2} \mathrm{Te}_{3}$ calculated with NEMD after the extrapolation for an infinite system size as a function of temperature.

\subsection{Substitution defects}

After calculating the thermal conductivity of bulk $\mathrm{Bi}_{2} \mathrm{Te}_{3}$, several stoichiometries of monocrystalline $\mathrm{Bi}_{40-\mathrm{x}} \mathrm{Te}_{60}(\mathrm{x}=1$ to 10$)$ and $\mathrm{Bi}_{40} \mathrm{Te}_{60-\mathrm{y}}(\mathrm{y}=1$ to 6$)$ have been studied, which are $\mathrm{n}$ and p-type respectively (Figure 3). The structures with percentages of vacancies of Te atoms greater than $7 \%$ were unstable. The purpose is to find the stoichiometries with vacancy defects yielding the lowest thermal conductivity or with the highest ZT. For the defected systems, only the $8 \times 8 \times 4$ structures were used to compute an effective thermal conductivity. The interest in the second case was to detect the impact of vacancy and defects on the thermal conductivity in comparison to the thermal conductivity of bulk $\mathrm{Bi}_{2} \mathrm{Te}_{3}$. Thermal conductivity for both $\mathrm{Bi}$ and Te vacancy defected structures indeed decreases in comparison to the thermal conductivity of stoichiometric $\mathrm{Bi}_{2} \mathrm{Te}_{3}$. The vacancy defects 
drive thermal conductivity to decrease more than $60 \%$ for $5 \%$ of Bismuth vacancy concentration and more than $70 \%$ for $4 \%$ of Tellurium vacancy concentration. In overall, we can mention that the vacancy defects destroy the continuity of the lattice and constitutes an additional scattering mechanism for phonons. The reduction of the thermal conductivity is very important, which is a promising direction for the increase of the figure of merit.

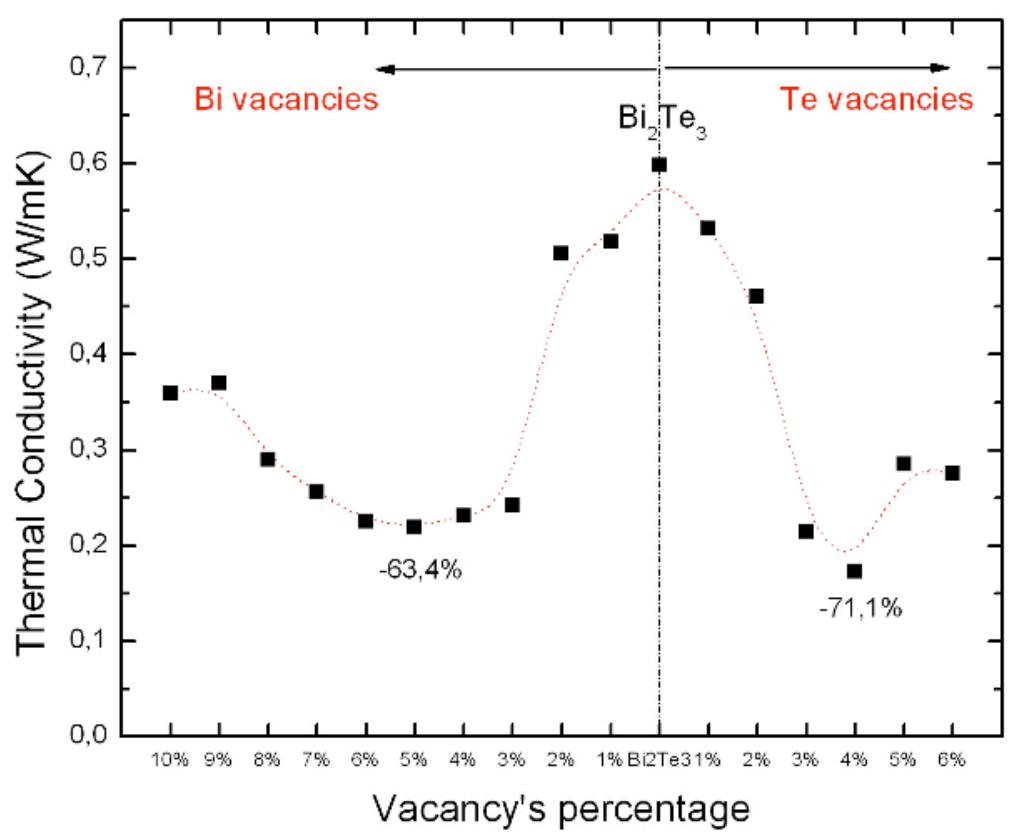

Fig. 3. Thermal conductivity for vacancy defected structures calculated with NEMD as a function of the vacancy concentration.

\section{Conclusions and Discussion}

With the current theoretical study, we achieved important reduction of the thermal conductivity of bulk $\mathrm{Bi}_{2} \mathrm{Te}_{3}$ by introducing of point defects into the lattice. The thermal conductivity can be decreased even by $70 \%$ for a small amount of vacancies of Tellurium atoms. The creation of superlattices with defected structures that exhibit the lowest known thermal conductivities are in the next plans. We expect further reduction of the thermal conductivity due to phonon confinement effects (introduction of interfaces). Furthermore the electrical conductivity and the Seebeck coefficient will be studied. We believe that the vacancy defects will not deteriorate the crystal like behaviour of the structure for electrons.

\section{References}

1. S. Kotake and S. Wakuri, JSME Int. J., Ser. B 37, 103 (1994)

2. R. Venkatesh, J. Amrit, Y. Chalopin and S. Volz, Phys. Rev. B, 83, 115425 (2011).

3. K. Termentzidis, P. Chantrenne and P. Keblinski, Phys. Rev. B 79, 214307 (2009)

4. K. Termentzidis et al, Nanoscale Research Letters 6, 288 (2011) 


\section{$2^{\text {nd }}$ European Energy Conference}

5. C. Abs-Da-Cruz, K. Termentzidis, P. Chantrenne and X. Kleber, J. Appl. Phys. 110, 034309 (2011)

6. K. Termentzidis, P. Chantrenne, S. Merabia and P. Keblinski, Int. J. Jeat and Mass Transfer 54, $2014(2011)$

7. K. Termentzidis, P. Chantrenne, J-Y. Duquense and A. Saci, J. Phys. : Cond. Matter 22, 475001 (2011)

8. S. Volz, J.B. Saulnier, G. Chen and P. Beauchamp, High Temperatures, High Pressures 32, 631 (2000)

9. S. Volz et al, Phys. Rev. B 54, 340 (1996)

10. B. Qiu and X. Ruan, Phys. Rev. B 80, 165203 (2009)

11. P. Schelling, S. Phillpot and P. Keblinski, Phys. Rev. B 65, 144306 (2002)

12. H.J. Goldsmid, Proc. Phys. Soc. B 69203 (1956)

13. C.B. Satterthwaite, and R. Ure, Phys. Rev. 108, 1164 (1957) 